\title{
Synthesis and characterization of thorium, uranium and cerium oxide nanoparticles
}

\author{
By O. N. Batuk ${ }^{1}$, D. V. Szabó ${ }^{2}$, M. A. Denecke ${ }^{3}$, T. Vitova ${ }^{3}$ and S. N. Kalmykov ${ }^{1, *}$ \\ ${ }^{1}$ Chemistry Department of Lomonosov Moscow State University, Moscow, Russia \\ 2 Forschungszentrum Karlsruhe GmbH, Institut für Materialforschung III, Karlsruhe, Germany \\ ${ }^{3}$ Forschungszentrum Karlsruhe GmbH, Institut für Nukleare Entsorgung, Karlsruhe, Germany
}

(Received July 12, 2011; accepted in final form July 14, 2012)

(Published online March 18, 2013)

\section{Actinide oxides / Nanoparticle / Mesoporous silica / Ultrasound}

Summary. We describe the synthesis of cerium, thorium and uranium oxide nanoparticles embedded in a mesoporous matrix as template in a kind of nanocasting technique. The solid matrix is used as a template to obtain and stabilize the actinide oxide nanoparticles. We apply high resolution transmission electron microscopy (HR-TEM) to show evidence of metal oxide incorporation into the matrix pores and analyze their structure. Measured interplanar distances and calculated lattice parameters for synthesized nanosized $\mathrm{CeO}_{2-x}$ and $\mathrm{ThO}_{2}$ samples differ from their bulk crystalline counterparts. We obtain with our synthesis $\mathrm{CeO}_{2-x}$ particles containing both $\mathrm{Ce}^{4+}$ and larger sized $\mathrm{Ce}^{3+}$. The lattice parameter for these ceria nanoparticles is found to be larger than the bulk value due to the presence of $\mathrm{Ce}^{3+}$ with its larger ionic radius. The presence of $\mathrm{Ce}^{3+}$ was established by means of high resolution X-ray emission spectroscopy (HRXES), applied to the investigation of nanoparticles for the first time. The $\mathrm{ThO}_{2}$ nanoparticles exhibit a decrease in interplanar distances, as one might generally expected for these nanoclusters. However, the lattice distance decrease for our particles is remarkable, up to $5 \%$, indicating that contact with the surrounding silica matrix may exert a bond distance shortening effect such as through significant external pressure on the particle surface.

\section{Introduction}

Investigation of nanoparticles is significant to our understanding of desirable, as well as undesirable, environmental, technical and basic solid state processes, in order to be able to control or predict their formation and stability. The evolution of a condensed solid often occurs via formation and agglomeration of nanoparticles. Nanoparticles are generally not stable, due to the reactivity of their high surface area, and therefore have a tendency to agglomerate into larger particles. Scientists and engineers dealing with nanotechnology are faced with the challenge of preparation and stabilization of nanoparticles in various media. Under the conditions of proposed spent nuclear fuel (SNF) or high level waste (HLW) repositories, where

\footnotetext{
*Author for correspondence (E-mail: stepan@ @adio.chem.msu.ru).
}

elevated radiation and thermal fields will prevail, formation of various nanoparticles have been demonstrated in several studies. Utsunomiya et al. [1] observed the formation of uraninite $\left(\mathrm{UO}_{2+x}\right)$ nanoparticles upon irradiation of boltwoodite, $(\mathrm{K}, \mathrm{Na})\left[\left(\mathrm{UO}_{2}\right)\left(\mathrm{SiO}_{3} \mathrm{OH}\right)\right]_{2}\left(\mathrm{H}_{2} \mathrm{O}\right)_{1.5}$, by heavy ions and in-situ high resolution transmission electron microscopy (HR-TEM) measurements. In our previous studies [2], we demonstrate the formation of schoepite, $\left[\left(\mathrm{UO}_{2}\right)_{8} \mathrm{O}_{2}(\mathrm{OH})_{12}\right]\left(\mathrm{H}_{2} \mathrm{O}\right)_{12}$, nanocrystals on a $\mathrm{UO}_{2+x}$ powder sample surface upon hydrothermal leaching at $150{ }^{\circ} \mathrm{C}$. The corrosion of canisters for SNF or HLW with the formation of green rust may lead to the reduction of U(VI) in solution, with subsequent precipitation of uraninite nanoparticles as demonstrated by O'Loughlin et al. [3].

Tetravalent actinides, An(IV), and their analogues, as highly hydrolyzable cations, have a strong tendency to polymerize. The formation of $\mathrm{Zr}(\mathrm{IV})$ [4], Th(IV) [5] and $\mathrm{Pu}(\mathrm{IV})$ [6] polymers in solution has been extensively studied $[7,8]$. According to high resolution nano-electrospray mass spectrometry results, the hydrolysis of polynuclear An(IV) species is a continuous process and upon exceeding the solubility limit An(IV) eigencolloids are formed. Associated investigations using extended X-ray absorption fine structure (EXAFS) and laser induced breakdown detection (LIBD) have allowed development of a model for $\mathrm{Pu}(\mathrm{IV})$ eigencolloid formation, which includes condensation of $\mathrm{Pu}_{n} \mathrm{O}_{p}(\mathrm{OH})_{4 n-2 p}\left(\mathrm{H}_{2} \mathrm{O}\right)_{z}$ oligomers [7]. Rothe et al. [8] reported that the white line intensity in X-ray absorption near edge structure (XANES) spectra of An(IV) eigencolloids depends on the degree of condensation of An(IV). To corroborate this finding well-defined An(IV) nanoparticles with a defined size and narrow size distribution should be investigated. To this aim, we have developed a method for synthesizing actinide oxide nanoparticles with defined and variable size.

The goal of our research here is to synthesize thorium, uranium and cerium oxide nanoparticles and their characterization. We consider thorium dioxide as a simple model for other actinide dioxide compounds, since it has the same fluorite-type crystal structure, is non-redox sensitive and does not form non-stoichiometric oxides. Uranium oxide is the main component of nuclear fuel and we synthesize uranium oxide nanoparticles as models for eigencolloids of 
this material. Cerium oxide is isostructural with thorium and uranium oxide but non-radioactive and therefore used to develop and refine the nanoparticle synthesis. We use an optimized method of oxide nanoparticle preparation and stabilization by embedding them into the pore structure of mesoporous silica as a solid template. This allows us to obtain particles with a narrow size distribution and to stabilize them against agglomeration. To study the relationship between particle size and structure, mesoporous matrixes of MCM-41 and SBA-15 families are used to synthesize particles with a mean size of 2, 3 and $5 \mathrm{~nm}$, which are then characterized by means of HR-TEM.

\section{Experimental section}

\subsection{Synthesis of mesoporous solid templates}

Mesoporous silica templates having the hexagonal pore structure of MCM-41 and SBA-15 silica families are synthesized as described previously [9-11]. The scheme of synthesis is schematically presented in Fig. 1. Briefly, the mesoporous silica synthesis is based on tetraethyloxysilane (TEOS) hydrolysis around micelles of surfactant (cetyltriammounium chloride, $\mathrm{CTACl}$ ) and triblock copolymer (poly(ethylene oxide)-poly(propylene oxide)-poly(ethylene oxide), P123) in aqueous alkaline media for MCM-41 and in aqueous acidic solution for SBA-15. Cylindrical wire shaped micelles are obtained at room temperature for $\mathrm{CTACl}$ and at $90^{\circ} \mathrm{C}$ for $\mathrm{P} 123$. To increase the diameter of CTACl micelles, trimethylbenzene (TMB) is used as a swelling reagent. The following molar ratios of reagents are used for the silica matrix synthesis: $1 \mathrm{TEOS} / 0.13 \mathrm{CTACl} / 5 \mathrm{NH}_{3} / 140 \mathrm{H}_{2} \mathrm{O}$ for $\mathrm{MCM}-41$ with pore size $2 \mathrm{~nm}$; 1TEOS/0.13CTACl/0.9TMB/ $5 \mathrm{NH}_{3} / 140 \mathrm{H}_{2} \mathrm{O}$ for MCM-41 with pore size $3 \mathrm{~nm} ; 1 \mathrm{TEOS} /$ $0.017 \mathrm{P} 123 / 5.7 \mathrm{HCl} / 193 \mathrm{H}_{2} \mathrm{O}$ for $\mathrm{SBA}-15$ with $5 \mathrm{~nm}$ pore size. The suspensions are stirred $30 \mathrm{~min}$ after all reagents are added. The solid and aqueous phases are then separated by filtration and washed with MilliQ water. The mesoporous silica are dried for $24 \mathrm{~h}$ at $60{ }^{\circ} \mathrm{C}$ and then annealed to remove organic surfactant material using the following regime: heating up to $550{ }^{\circ} \mathrm{C}$ with ramping $1{ }^{\circ} \mathrm{C} / \mathrm{min}$, calcination for $12 \mathrm{~h}$ and subsequent cooling down to room temperature with the same ramping $1{ }^{\circ} \mathrm{C} / \mathrm{min}$ rate. The internal pore diameters of the synthesized silica templates are determined using an Autosorb-1 (Quantachrome KG, Germany). We measure $\mathrm{N}_{2}$ sorption and desorption at $77 \mathrm{~K}$ and evaluate data with the BET method to calculate free surface area and the BJH (Barrett-Joyner-Halenda) method to calculate pore size distribution to be approximately 2 and $3 \mathrm{~nm}$, in the case of MCM-41, and $5 \mathrm{~nm}$ for SBA-15 (see Table 1).

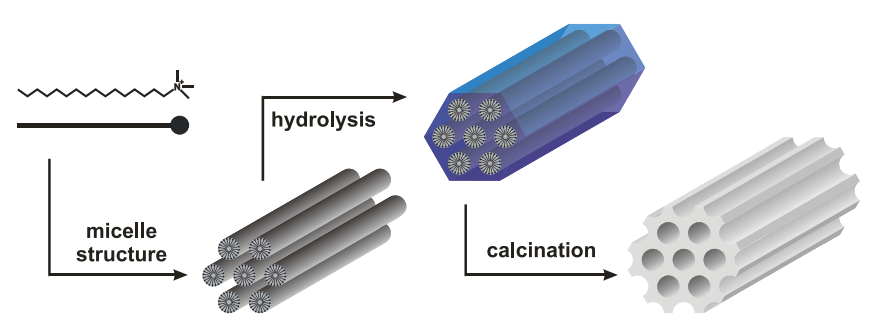

Fig. 1. Scheme of mesoporous silica synthesis based on TEOS hydrolysis in the presence of surfactant material micelles.
Table 1. The pore size of synthesized mesoporous silica templates determined from HR-TEM images and from BJH-analysis.

\begin{tabular}{lcc}
\hline Matrix & TEM fringes, nm & Pore diameter, nm (BJH) \\
\hline MCM-41 (1) & 2.9 & 2.0 \\
MCM-41 (2) & 4.7 & 3.2 \\
SBA-15 & 9.5 & 4.8 \\
\hline
\end{tabular}

HR-TEM is used to characterize the synthesized mesoporous materials. HR-TEM investigations are performed using a TECNAI F20 ST instrument (FEI Company, USA), operating at $200 \mathrm{kV}$. We prepare samples for microscopy by dropping sample suspensions in water onto a copper grid covered by Lacey carbon film and subsequent drying in air. The micrograph images show the regularity of the pore structure for the synthesized materials and the distance of the fringes are measured to be larger than inside pore diameter values and those determined with BJH (Table 1). The measured fringes are larger compare to pore diameters determined by $\mathrm{BJH}$ due to the fact that HR-TEM gives a lattice parameter of mesoporous structure, considering pores themselves and walls in between.

\subsection{Synthesis of Ce, Th and $U$ oxide nanoparticles}

To embed actinide oxide nanoparticles into the mesoporous pore structure, we treat the mesoporous template with aqueous solutions containing $\mathrm{Ce}(\mathrm{III}), \mathrm{Th}(\mathrm{IV})$ or $\mathrm{U}(\mathrm{VI})$ at a total metal ion concentration of $5 \times 10^{-3} \mathrm{M}$. We use $\mathrm{Ce}(\mathrm{III})$ nitrite solution as a precursor for $\mathrm{CeO}_{2}$ nanoparticles due to the instability of $\mathrm{Ce}(\mathrm{IV})$ compounds in solution. Th(IV) and U(VI) chloride solutions are added as precursor. We prepare samples under air; no special gas atmosphere is used. In order to facilitate metal ion entry into the MCM-41 pore structure, the suspension of silica and dissolved metal ions is subjected to ultrasonic treatment at a frequency of $35 \mathrm{kHz}$. The starting $\mathrm{pH}$ values of suspensions in the both sets of matrices are $\mathrm{pH} 1$ for $\mathrm{Th}(\mathrm{IV}), \mathrm{pH} 2$ for $\mathrm{U}(\mathrm{VI})$ and $\mathrm{pH} 6$ for $\mathrm{Ce}(\mathrm{III})$, with stepwise increase to $\mathrm{pH}$ values of $3.5,4.5$ and 8.5 for $\mathrm{Th}, \mathrm{U}$ and $\mathrm{Ce}$, respectively. The $\mathrm{pH}$ values of the suspensions are adjusted using $\mathrm{NaOH}$ and $\mathrm{HCl}$ solutions with various concentrations. After ultrasonic treatment or magnetic stirring (for $\sim 5 \mathrm{~nm}$ particles in the SBA-15 template), the solid material is filtered, washed with MilliQ water to remove any surface precipitates and then heated in air with a $30{ }^{\circ} \mathrm{C} / \mathrm{min}$ ramping rate to $250{ }^{\circ} \mathrm{C}$ and tempered for a duration of $2 \mathrm{~h}$. HR-TEM is used to characterize the synthesized mesoporous material prior to and following nanoparticle preparation.

\subsection{Spectroscopic characterization of the nanoparticles}

X-ray absorption near edge structure (XANES) spectroscopy is applied on uranium oxide nanoparticles at the INEBeamline for actinide research at the ANKA synchrotron, Karlsruhe, Germany. The spectra are recorded at $L_{3}$ uranium edge $(17178 \mathrm{eV})$ in fluorescence mode (5 element 
germanium solid-state detector, Canberra). The beam is monochromatized using a $\mathrm{Si}(111)$ crystal pair in the double crystal monochromator (DCM). The DCM energy is calibrated to the $\mathrm{Y}$ foil $1 \mathrm{~s}$ ionization energy of $17038 \mathrm{eV}$, which is assigned to the first inflection point in the first derivative of the Y foil reference foil spectrum. A schoepite sample as uranium standard is mounted behind the uranium oxide nanoparticle samples and measured simultaneously. Up to six scans are averaged to improve the signal-to-noise ratio of the measurements.

High resolution X-ray emission spectroscopy (HRXES) experiments are performed on the $2 \mathrm{~nm} \mathrm{CeO}$ nanoparticles at the BL-W1 experimental station at HASYLAB, Hamburg, Germany. The $L_{\alpha 1}$ fluorescence emitted by the sample following excitation of $2 p_{3 / 2}$ core states using radiation coming from the wiggler at BL-W1 is energy dispersed using a $\mathrm{Si}(400)$ analyzer crystal, at Bragg angle of $70.66^{\circ}$. The crystal with a $1 \mathrm{~m}$ bending radius focuses the dispersed emission onto a CCD detector (Princeton Instruments, USA). The wavelength of the incident excitation radiation is monochromatized using a $\mathrm{Si}(111)$ crystal pair in the DCM. Spectra are recorded by registering the emission signal for each incident energy increment $1,0.5$, 2 and $3 \mathrm{eV}$ for energy regions 5700-5713, 5713.5-5752, $5744-5760$ and $5763-5787 \mathrm{eV}$, respectively. The integral emission intensity at $4.830 \pm 0.001 \mathrm{keV}$ for each point, normalized to the incident intensity measured with an airfilled ionization chamber, is plotted to obtain high resolution XANES.

\section{Results and discussion}

Typical HR-TEM images of the mesoporous silica are shown in Fig. 2. All mesoporous structures are observed to be sensitive to irradiation by the electron beam. Fig. $2 \mathrm{a}$ shows the microstructure of mesoporous MCM-41 with nominally $2 \mathrm{~nm}$ sized pores, Fig. $2 \mathrm{~b}$ with $3 \mathrm{~nm}$ pores, and Fig. $2 \mathrm{c}$ the structure of SBA-15 with $5 \mathrm{~nm}$ pores.

Representative high-resolution TEM images of the prepared oxide nanoparticles embedded in mesoporous MCM41 are presented in Fig. 3. Fig. 3a shows nanoparticles of $\mathrm{ThO}_{2}$ and Fig. $3 \mathrm{~b}-\mathrm{CeO}_{2}$. As seen in Fig. 3, the pore structure of the mesoporous silica is apparently destroyed during metal ion impregnation and subsequent annealing. In a blank experiment, without annealing, the ultrasound treatment is observed to have no effect on MCM-41 pore structure.

We find that ultrasonic treatment plays a critical role in getting nanoparticle to form inside of the pore structure of the MCM-41 matrix [12]. Upon application of conventional stirring, without ultrasonic treatment of the suspensions, only hydroxide precipitates are observed to form on the external MCM-41 surfaces, with no embedding into the silica pore structure. Using the ultrasonic treatment, the embedded nanoparticles in the pores are distributed homogeneously over the silica matrix as, for example, demonstrated by the bright field STEM image of U(VI) oxide nanoparticles (Fig. 4a). The nanoparticles are seen as black spots, originated by $Z$-contrast, distributed throughout the grey region, corresponding to the silica matrix. EDX analysis con-

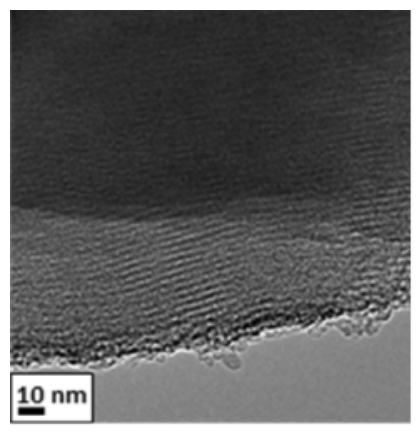

(a)

Fig. 2. HR-TEM images of mesoporous silica templates after annealing: (a) $2 \mathrm{~nm}$ (MCM-41), (b) $3 \mathrm{~nm}$ (MCM-41) and (c) $5 \mathrm{~nm}$ (SBA-15).

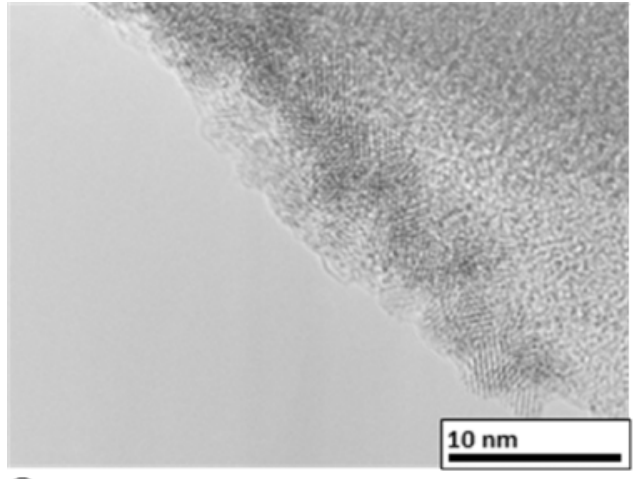

(a)

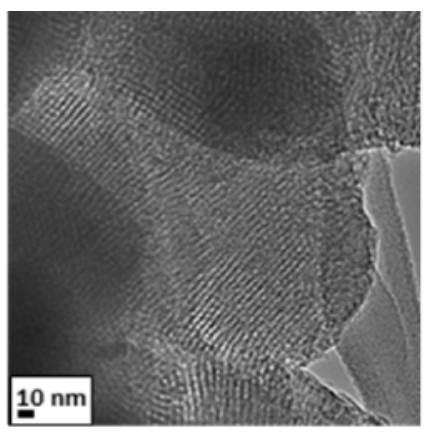

(b)

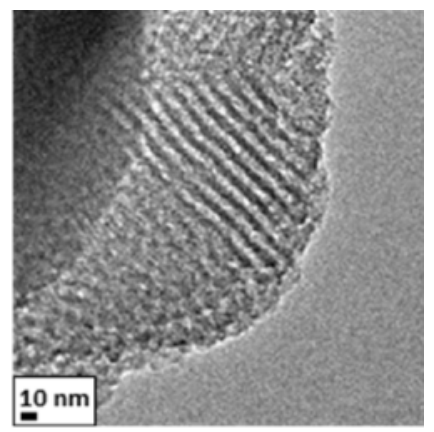

(c)

Fig. 3. HR-TEM images of $3 \mathrm{~nm}$ nanoparticles (spots with grids) embedded into mesoporous MCM-41 matrix: (a) thorium dioxide, (b) cerium dioxide. 


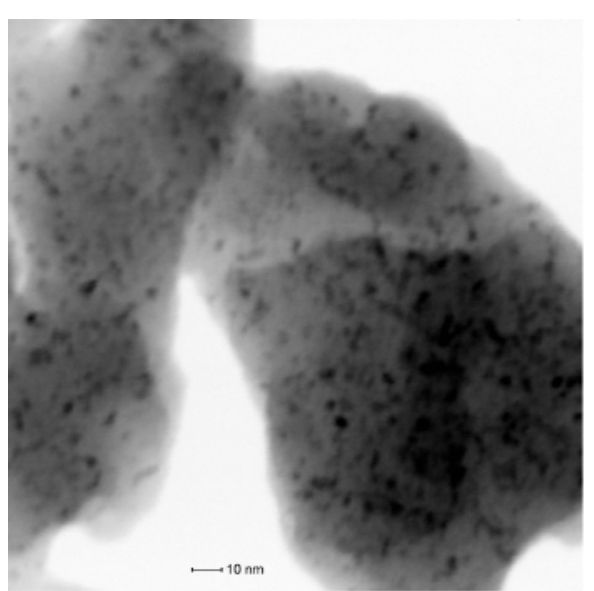

(a)

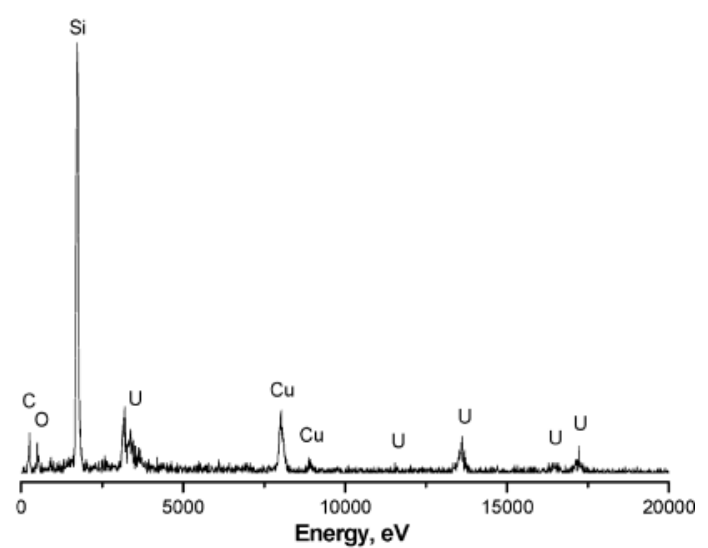

(b)

Fig. 4. (a) Bright field STEM image of uranium nanoparticles embedded into mesoporous matrix. Uranium oxide nanoparticles are distinguished as dark spots in the grey background corresponding to the silica matrix. (b) EDX-spectrum collected at the area shown in (a).

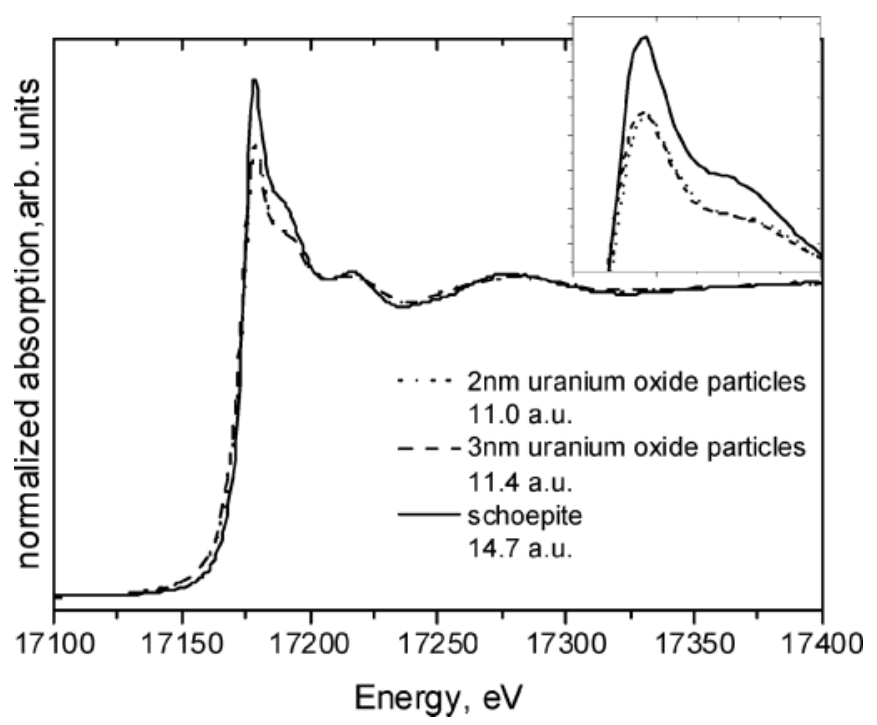

Fig. 5. $\mathrm{U} L_{3}$ XANES spectra for uranium oxide nanoparticles and schoepite. The white line area is estimated using Gauss function (indicated in arbitrary units). The insert shows a zoom of the white line maximum between $17170 \mathrm{eV}$ and $17200 \mathrm{eV}$.

firmed that the spots contain U (Fig. 4b). The carbon and copper signals on EDX spectra are from the grid, oxygen signal is low due to self absorption.

The valence of uranium in the structure of oxide nanoparticles is analyzed in-situ by XANES method. The $\mathrm{U} L_{3}$ XANES spectra are presented in Fig. 5. The energy position and the shape of the spectra is comparable to the reference schoepite spectrum and indicate the presence of $\mathrm{U}(\mathrm{VI})$ in the nanoparticles. The white line decrease is associated with a line broadening, as well as the white line area (indicated in arbitrary units (a.u.) in Fig. 5) decrease over that for schoepite. The decrease in intensity is interpreted as a decrease in $6 d$-like final state density involved in the $2 p_{3 / 2} \rightarrow 6 d$ dipole allowed $L_{3}$ edge transition for the nanoparticles. We note that the observed intensity decrease from the bulk schoepite, to $3 \mathrm{~nm}$ particles, to $2 \mathrm{~nm}$ particles (see Fig. 5, insert) is also associated with the reduction in bulk/surface ratio, i.e. increasing significance of surface states for smaller sized particles. An increase in surface sites over bulk is equivalent to a decrease in the overall average structural order. This disorder can lead to final state broadening, associated decrease in state density, and, hence, lowering of the $2 p_{3 / 2} \rightarrow 6 d$ transition probability and white line intensity.

We determine the lattice parameter of our synthesized cerium and thorium oxide nanoparticles of varying sizes from measurement of two different d-spacings in HRTEM profiles for 5-16 different individual $\mathrm{ThO}_{2}$ nanoparticles and 6-9 $\mathrm{CeO}_{2}$ nanoparticles. The results are presented in Table 2 and compared to values for bulk metal oxides $[13,14]$. The lattice parameter calculated from (111) spacings for the $\mathrm{CeO}_{2}$ nanoparticles (Table 2) are all larger than those from the (200) interplanar distances. All the $a$-values are larger than the bulk $\mathrm{CeO}_{2}$ value with one exception, that calculated for $2 \mathrm{~nm}$ nanoparticles using the (200) spacing; this value is associated with the largest error, however. In contrast, the lattice spacings and parameters for all $\mathrm{ThO}_{2}$ nanoparticles studied are significantly smaller compared to the bulk phase values. The average lattice parameter is $5.3 \AA$, which is $5 \%$ smaller than the bulk value, and there is a trend toward longer lattice parameters with increasing particle size.

Several papers have reported that the structure of metal or metal oxide nanoparticles, i.e. their lattice parameters, can differ from those of the bulk phase [15-26]. For metal nanoparticles, a decrease in lattice parameter is compared to bulk values is often observed [15-17]. This is generally explained by internal pressure produced by surface stress in small particles. For some metal oxide nanoparticles, an inverse phenomenon has been reported: the lattice parameters are in these cases observed to be larger than those for their bulk phases [18-22]. This is explained by the compensation of surface charge of oxide nanoparticles through an increase in the distances between atoms.

The surface free energy of small particles plays an important role in particle size, as well as surface tension, reactivity, and shape. Qi and Wang [15], e.g., proposed a model for the various size and shapes of metal nanoparticles based on calculation of free surface energy (minimization of its 
Table 2. Interplanar distances in nanoparticles from HR-TEM images for the crystallographic planes indicated and corresponding calculated lattice parameters, compared to values for the bulk phases. Values in parentheses give estimated errors of the determination.

\begin{tabular}{|c|c|c|c|c|c|}
\hline \multirow{2}{*}{$\begin{array}{l}\text { Type and number } \\
\text { of particles } \\
\mathrm{CeO}_{2} / \mathrm{MCM}-41\end{array}$} & \multicolumn{2}{|c|}{$\begin{array}{l}\text { Distances and lattice parameter }(a) \\
\text { for nanoparticles, }[\AA]\end{array}$} & \multicolumn{3}{|c|}{$\begin{array}{l}\text { Distances and lattice parameter }(a) \\
\text { for bulk phase, }[\AA]\end{array}$} \\
\hline & $3.20(2)(111)$ & $a=5.54(3)$ & \multirow[t]{6}{*}{$\mathrm{CeO}_{2}$} & $3.127(111)$ & $a=5.4124(1)$ \\
\hline $2 \mathrm{~nm} 6$ particles & $2.70(10)(200)$ & $a=5.40(10)$ & & $2.708(200)$ & \\
\hline $\mathrm{CeO}_{2} / \mathrm{MCM}-41$ & $3.21(2)(111)$ & $a=5.56(3)$ & & & \\
\hline $3 \mathrm{~nm} 9$ particles & $2.72(5)(200)$ & $a=5.44(9)$ & & & \\
\hline $\mathrm{CeO}_{2} / \mathrm{SBA}-15$ & $3.21(1)(111)$ & $a=5.56(2)$ & & & \\
\hline $5 \mathrm{~nm} 6$ particles & $2.75(3)(200)$ & $a=5.50(10)$ & & & \\
\hline $\mathrm{ThO}_{2} / \mathrm{MCM}-41$ & $3.03(7)(111)$ & $a=5.30(10)$ & \multirow[t]{6}{*}{$\mathrm{ThO}_{2}$} & $3.233(111)$ & $a=5.6001(3)$ \\
\hline $2 \mathrm{~nm} 9$ particles & $2.65(6)(200)$ & $a=5.30(10)$ & & $2.800(200)$ & \\
\hline $\mathrm{ThO}_{2} / \mathrm{MCM}-41$ & $3.05(7)(111)$ & $a=5.28(3)$ & & & \\
\hline $3 \mathrm{~nm} 16$ particles & $2.63(4)(200)$ & $a=5.26(8)$ & & & \\
\hline $\mathrm{ThO}_{2} / \mathrm{SBA}-15$ & $3.07(3)(111)$ & $a=5.32(5)$ & & & \\
\hline $5 \mathrm{~nm} 5$ particles & $2.67(3)(200)$ & $a=5.34(6)$ & & & \\
\hline
\end{tabular}

Gibbs energy). Their calculations showed that lattice parameter decrease with decreasing particle size. Such trends apparently work for particles larger than $1 \mathrm{~nm}$, but not for wires or thin films. The model also showed that the influence of nanoparticle shape on lattice parameter contributes not more than $10 \%$ to the total deviation from the bulk values; the main parameter influencing lattice parameter is size. The model of Qi and Wang was successfully tested experimentally using Pd clusters $1.4-5 \mathrm{~nm}$ in diameter, where both theory and experiment revealed a decrease in lattice parameter of up to $2.5 \%$ compared to the bulk material. The lattice parameter decrease is the result of surface stress, itself a function of particle size, and leads to the observed decrease in crystal plane distances. The same effect was observed for a $\mathrm{Al} / \mathrm{MgO}$ sandwich system [17], where the lattice parameter for particles smaller than $20 \mathrm{~nm}$ in diameter was observed to decrease nearly $2 \%$ for the smallest particles $(6 \mathrm{~nm})$. For particles with diameters less then $8 \mathrm{~nm}$, the decrease in interatomic distances was found to be a function of the distance from the particle centre.

The lattice parameter dependency on nanoparticle size has been investigated for a number of oxide systems such as $\mathrm{CeO}_{2} / \mathrm{CeO}_{2-x}$ and $\mathrm{TiO}_{2}$, which have the same cubic crystal structure as our nanoparticles, and $\mathrm{Al}_{2} \mathrm{O}_{3}, \mathrm{Fe}_{2} \mathrm{O}_{3}, \mathrm{PbTiO}_{3}$, $\mathrm{PbZrO}_{3}$, which as partially covalent oxides tend to exhibit a transformation of their crystal structure to higher symmetry as small particles [18]. Previously published studies of $\mathrm{CeO}_{2}$ and $\mathrm{TiO}_{2}$ nanoparticles demonstrate that the lattice parameter increases with decreasing particle size [19-22]. This is explained by the formation of charge defects, the concentration of which is inversely proportional to size. Metal cation charge compensates for the defects, in this case $\mathrm{Ce}^{3+}$, which contributes to systematic lattice expansion in small particles (smaller than $20 \mathrm{~nm}$ ) because of its larger ionic radius and due to its decreasing the electrostatic attraction between ions.

These literature studies help identify reasons behind the contrasting trends in particle size and lattice distances between the $\mathrm{CeO}_{2}$ and $\mathrm{ThO}_{2}$ nanoparticles. Assuming that the synthesized $\mathrm{CeO}_{2}$ nanoparticles behave similarly as in previous reports, e.g. [21], in that their increased lattice parameters are larger due to formation of charge defects (oxygen vacancies) and compensation with $\mathrm{Ce}^{3+}$, we attempt to dis- cern if our nanoparticles indeed contain $\mathrm{Ce}^{3+}$. To this end we record Ce $L_{3}$ HRXES spectra of bulk $\mathrm{CeO}_{2}$ and compare this to our $2 \mathrm{~nm} \mathrm{CeO}$ nanoparticles. This is a similar strategy to using $\mathrm{Ce} L_{3} \mathrm{X}$-ray absorption near-edge structure (XANES) analysis [23], but by monitoring the partial fluorescence yield, thereby circumventing life-time spectral broadening, near-edge spectra with much better resolution are obtained. The spectra are depicted in Fig. 6. The bulk $\mathrm{CeO}_{2} L_{3}$ HRXES exhibits a splitting of the white line, also observed in its conventional XANES [24], due to introduction of multiple final states through hybridization between $\mathrm{O} 2 p$ and $\mathrm{Ce} 4 f(5 d)$ states. These spectral features in the HRXES are significantly better resolved, however. The HRXES for the nanoparticle sample is different than the bulk spectrum; it shows evidence for the presence of $\mathrm{Ce}^{3+}$ along with the $\mathrm{Ce}^{4+}$. The spectrum exhibits a relatively higher intensity for the low energy feature. The feature itself is shifted to lower energy by nearly $3 \mathrm{eV}$, which is the expected energy shift of the main absorption maximum of $\mathrm{Ce}^{3+}$ relative to $\mathrm{Ce}^{4+}$ [23]. This spectroscopic evidence of the presence of both $\mathrm{Ce}^{3+}$ and $\mathrm{Ce}^{4+}$ in the nanoparticles supports the interpretation of their increased lattice constant being

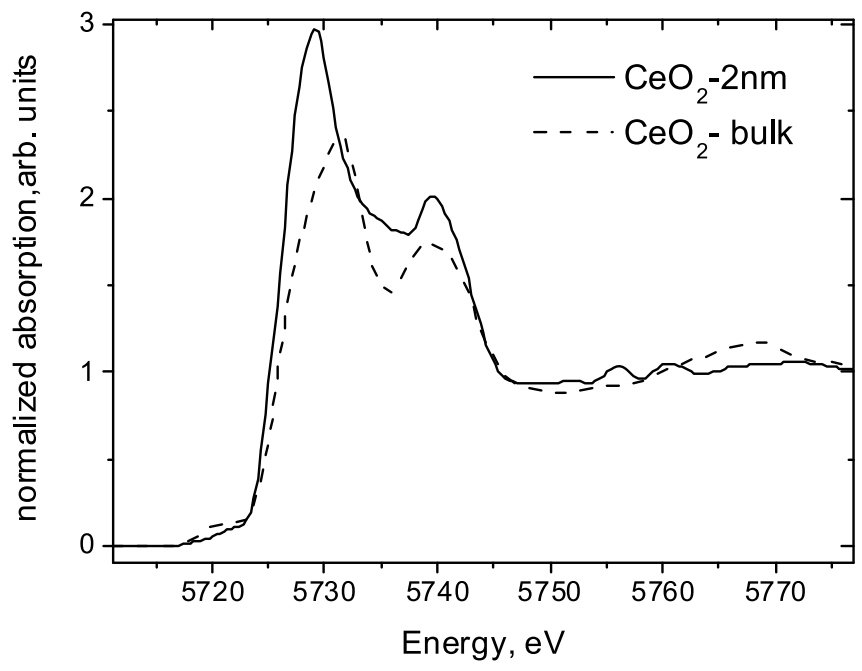

Fig. 6. Ce $L_{3}$ HRXES near edge spectra recorded for the $2 \mathrm{~nm}$ ceria nanoprticles embedded in MCM-41 (solid line) and bulk $\mathrm{CeO}_{2}$ (dashed line). 
due to $\mathrm{Ce}^{3+}$ cation and oxygen vacancy charge defects to compensate surface free energy changes caused by decreasing particle size. Our nanoparticles may be better described as $\mathrm{CeO}_{2-x}$. This is similar to results previously reported for nano-ceria in, e.g. [24].

In contrast to the $\mathrm{CeO}_{2}$ nanoparticles, the $\mathrm{ThO}_{2}$ nanoparticles exhibit a trend towards shortening of interplanar distances with decreasing particle size. This contrasting behavior can partially be explained by the fact that Th(IV), as opposed to cerium, is redox insensitive; thorium nominal valence does not change, except under extreme conditions. In this case, changes in surface forces such as surface stress/charge as the particle size decreases cannot be compensated by introduction of any other valent Th/charge defects. In terms of near-ordered surface structure, nanoparticle surface $\mathrm{Th}^{4+}$ cations are likely oxygen terminated [25] and coordination number (CN) deficient. This generally is associated with bond contraction and a decrease in interplanar distances. Herein likely lies one factor affecting the observed decrease in lattice constant in our $\mathrm{ThO}_{2}$ nanoparticles. Obviously, interaction of the particle surface with the covalent silica oxide template host does not offset any decrease in interplanar distances caused by such bond contractions. Apparently the silica host adjacent to the particle affects an increase in the particle internal pressure, surface tension and/or overall electrostatic forces, thereby augmenting the decrease in interplanar distances. To support this hypothesis, we compare results reported for uranium nanoparticles embedded in a carbon matrix. From TEM examination of uranium contaminated fly ash, Utsunomiya et al. [26] reported decreased interatomic distances for uraninite nanoparticles embedded in fullerene-like carbonaceous material compared to those the bulk phase. In this case, interaction between carbonaceous material and the surface of embedded uranium dioxide nanoparticles also affects particle structure.

\section{Conclusion}

A method of metal oxide nanoparticle preparation is described, based on the impregnation of mesoporous silica pores, as nanocasting templates, with solutions containing these metal ions and subsequent calcinations. For pores smaller than $5 \mathrm{~nm}$, impregnation is effected by ultrasonic treatment. Using this method discrete crystalline Th(IV), $\mathrm{U}(\mathrm{VI})$ and $\mathrm{Ce}(\mathrm{IV})$ oxide nanoparticles, embedded in MCM41 and SBA-15 mesoporous silica matrices, are synthesized. The structure of thorium and cerium dioxide nanoparticles are observed to show contrasting differences in their interplanar distances compared to corresponding bulk phases; an increase is observed for the ceria and a decrease for the thoria nanoparticles. These differences lie in the metal cation nominal valence for the two systems. We obtain with our synthesis $\mathrm{CeO}_{2-x}$ particles containing both $\mathrm{Ce}^{4+}$ and larger sized $\mathrm{Ce}^{3+}$ leading to lengthening of the lattice constant of the nanoparticle crystallites. Our HR-TEM data do not allow us to discern any systematic increase in lattice parameter with decreasing particle size, however. For redox invariant Th(IV), bond contraction caused by dangling bonds and ten- sion on the particle surface leads to a decrease in lattice parameter. We propose that the silica matix used to template the nanoparticles affects additional surface forces contributing to relaxation of the interatomic distances inside the nanoparticle bulk.

This method of synthesis will be used for synthesizing well-defined nano-sized systems, making them available for investigations of their structural and spectroscopic properties. In particular, study of the electronic structure using both resonant inelastic scattering, a technique using the same experimental setup as HRXES yielding bulk information, combined with XPS, providing surface information, will help us identify differences in the structure of the particle surface $v s$. its center.

Acknowledgment. We acknowledge financial support from Russian Basic Research Foundation (contract 10-03-01029) and Ministry of Education and Scinece (contracts 02.740.11.0853 and 11.519.11.5011). We gratefully acknowledge T. Kisely (FZK INE) for the BET-analyses.

\section{References}

1. Utsunomiya, S., Wang, L. M., Ewing, R. C.: Radiation-induced decomposition of $\mathrm{U}(\mathrm{VI})$ alteration phases of $\mathrm{UO}_{2}$. Earth Planet. Sci. Lett. 240, 521 (2005).

2. Batuk, O. N., Kalmykov, St. N., Zakharova, E. V., Teterin, Yu. A.: Abstracts of the International Conference 37 Journees des Actinides Sesimbra, Portugal, 2007, 89-90 (2007).

3. O'Loughlin, E. J., Kelly, S. D., Cook, R. E., Csencsits, R., Kemner, K. M.: Reduction of uranium(VI) by mixed iron(II)/iron(III) hydroxide (green rust): formation of $\mathrm{UO}_{2}$ nanoparticles. Environ. Sci. Technol. 37, 721 (2003).

4. Cho, H. R., Walther, C., Rothe, J., Neck, V., Denecke, M. A., Dardenne, K., Fanghänel, Th.: Combined LIBD and XAFS investigation of the formation and structure of $\mathrm{Zr}(\mathrm{IV})$ colloids. Anal. Bioanal. Chem. 383, 28 (2005).

5. Walther, C., Fuss, M., Büchner, S.: Formation and hydrolysis of polynuclear Th(IV) complexes - a nano-electrospray massspectrometry study. Radiochim. Acta 7, 411 (2008).

6. Walther, C., Rothe, J., Altmaier, M., Marquardt, Ch., Fuss, M., Büchner, S.: NRC7 - seventh international conference on nuclear and radiochemistry 2008. Budapest, Hungary (2008).

7. Rothe, J., Walter, C., Denecke, M. A., Fanghaenel, Th.: XAFS and LIBD investigation of the formation and structure of colloidal $\mathrm{Pu}(\mathrm{IV})$ hydrolysis products. Inorg. Chem. 43, 4708 (2004).

8. Rothe, J., Denecke, M. A., Neck, V., Muller, R., Kim, J. I.: XAFS Investigation of the structure of aqueous thorium(IV) species, colloids, and solid thorium(IV) oxide/hydroxide. Inorg. Chem. 41, 249 (2002).

9. Beck, J. S., Vartuli, J. C., Roth, W. J., Leonowicz, M. E., Kresge, C. T., Schmitt, K. D., Chu, C. T-W., Olson, D. H., Sheppard, E. W., McCullen, S. B., Higgins, J. B., Schlenker, J. L.: A new family of mesoporous molecular sieves prepared with liquid crystal templates. J. Am. Chem. Soc. 114, 10834 (1992).

10. Grun, M., Unger, K. K., Matsumoto, A., Tsutsumi, K.: Novel pathways for the preparation of mesoporous MCM-41 materials: control of porosity and morphology. Micropor. Mesopor. Mat. 27, 207 (1999).

11. Yamada, T., Zhou, H., Asai, K., Honma, I.: Pore size controlled mesoporous silicate powder prepared by triblock copolymer templates. Mater. Lett. 56, 93 (2002).

12. Suslick, K. S.: Sonochemistry, 4th ed., Vol. 26, John Wiley and Sons, Inc., New York (1998).

13. Wolcyrz, M., Kepinski, L.: Rietveld refinement of the structure of $\mathrm{CeOCI}$ formed in $\mathrm{Pd} / \mathrm{CeO}_{2}$ catalyst: Notes on the existence of a stabilized tetragonal phase of $\mathrm{La}_{2} \mathrm{O}_{3}$ in $\mathrm{LaPdO}$ system. J. Solid State Chem. 99, 409 (1992).

14. Idiri, M., Le Bihan, T., Heathman, S., Rebizant, J.: Behavior of actinide dioxides under pressure: $\mathrm{UO}_{2}$ and $\mathrm{ThO}_{2}$. Phys. Rev. B 70, 014113 (2004). 
15. Qi, W. H., Wang, M. P.: Size and shape dependent lattice parameters of metallic nanoparticles. J. Nanopart. Res. 7, 51 (2005).

16. Lamber, R., Wetjen, S., Jaeder, N. I.: Size dependence of the lattice parameter of small palladium particles. Phys. Rev. B 51, 10968 (1995)

17. Woltersdorf, J., Nepijko, A. S., Pippel, E.: Dependence of lattice parameters of small particles on the size of the nuclei. Surface Sci. 106, 64 (1981).

18. Ayyub, P., Palkan, V. R., Chattopadnyay, S., Multani, M.: Effect of crystal size reduction on lattice symmetry and cooperative properties. Phys. Rev. B 51, 6135 (1995).

19. Tonej, A. M., Djerdj, I., Tonejc, A.: An analysis of evolution of grain size-lattice parameters dependence in nanocrystalline $\mathrm{TiO}_{2}$ anatase. Mat. Sci. Eng. C 19, 85 (2002).

20. Djerdj, I., Tonejc, A. M.: Structural investigations of nanocrystalline $\mathrm{TiO}_{2}$ samples. J. All. Comp. 413, 159 (2006).

21. Zhang, F., Chan, S. W., Spanier, J. E., Apak, E., Jin, Q., Robinson, R. D., Herman, I. P.: Cerium oxide nanoparticles: size- selective formation and structure analysis. Appl. Phys. Lett. 80, 127 (2002).

22. Wu, L., Wiesmann, H. J., Moodenbaugh, A. R., Klie, R. F., Zhu, Y., Welch, D. O., Suenaga, M.: Oxidation state and lattice expansion of $\mathrm{CeO}_{2-x}$ nanoparticles as a function of particle size. Phys. Rev. B 69, 125415 (2004).

23. Zhang, J., Wu, Z., Liu, T., Hu, T., Wu, Z., Ju, X.: XANES study on the valence transitions in cerium oxide nanoparticles. J. Synch. Rad. 8, 531 (2001).

24. Tsunekawa, S., Sivamohan, R., Ito, S., Kasuya, A., Fukuda, T.: Structural study on monosize $\mathrm{CeO}_{2-x}$ nano-particles. Nanostruct. Mat. 11, 141 (1999).

25. Rajh, T., Poluektov, O. I., Thurnauer, M. C.: Chemical Physics of Nanostructured Semiconductors. (Kokorin, A. I., Bahnemann, D. W., eds.) NOVA Science, New York (2003), Chapt. 1, pp. 1-34.

26. Utsunomiya, S., Jensen, K. A., Keeler, G. J., Ewing, R. C.: Uraninite and fullerene in atmospheric prticulates. Env. Sci. Tech. 36, 4943 (2002). 